\title{
Approche écologique du problème de la responsabilité de l'Homme et du Rat dans le fonctionnement d'un site de transmission à Scbistosoma mansoni en Guadeloupe
}

\author{
par A. THERON *, J.-P. POINTIER ** et C. COMBES * \\ * Département de Biologie animale, Centre Universitaire, avenue de Villeneuve, \\ F 56025 Perpignan Cedex. \\ 5aboratoire de Biologie marine et Malacologie, Ecole Pratique des Hautes Etudes, \\ 55, rue de Buffon, F 75005 Paris.
}

\section{Résumé.}

L'étude du taux de contamination des B. glabrata et des densités de cercaires de S. mansoni des canaux de la vallée de Beaugendre en Guadeloupe fait apparaitre la focalisation extrême des foyers à haut risque de contamination. Cette focalisation est directement liée à la répétition des rejets fécaux en des points déterminés. Un nombre limité de personnes contaminées suffit à maintenir des densités cercariennes élevées $(230 \mathrm{c} / 1)$. Dans le canal étudié, la responsabilité du Rat dans le fonctionnement du site apparaît moins importante que celle de l'Homme. L'infestation des rats pendant la consommation de planorbes est envisagée.

\section{Summary.}

An ecological approach to the problem of the responsability of men and rats in the workings of a transmission site of Schisiosoma mansoni in Guadeloupe (Western Indies).

The study of the rate of contamination of B. glabrata and the cercarial densities of $S$. mansoni in the chanels of Beaugendre valley (Guadeloupe, French West Indies) concentrates attention on the focalisation of the sites with high risk of contamination. This focalization of the sites of transmission is directly related to the regular deposition of human faeces. A small number of contamined people is enough to maintain high cercarial densities

Reçu le 17 octobre 1977. 
$(230 \mathrm{c} / \mathrm{l})$. In the chanel studied, the role of the rat in the transmission of the parasite seems less important than that of the Man. The infection of the rats during consumption of snails can be foreseen.

\section{Introduction}

Les recherches effectuées en Guadeloupe (1) depuis plusieurs années ont conduit à distinguer deux grands types de sites de transmission de la Schistosomose à Schistosoma mansoni :

- Les sites de transmission en milieu courant : essentiellement localisés dans la région montagneuse de l'île (Basse-Terre), ces sites de transmission sont représentés dans la plupart des cas par des canaux (canaux à usage domestique, d'irrigation ou d'évacuation des usines). Ces biotopes, liés à une importante activité de l'Homme, sont responsables en priorité de son infestation (Houin et al., 1973 ; Golvan et al., 1974);

- Les sites de transmission en milieu non courant : moins dispersés que les précédents, ils sont représentés par le Grand Etang en Basse-Terre et par la Mangrove palustre en Grande-Terre. Peu anthropisés, ces biotopes sont responsables en priorité d'une schistosomose murine (Combes et al., 1975; Nassi et al., 1975);

Dans un site de transmission en milieu courant, caractéristique de la Basse-Terre (Canal de Marigot) (2), nous avons recherché, par une approche quantitative et qualitative des principaux éléments du cycle (mollusques, cercaires, rats et hommes), à préciser : d'une part, le fonctionnement du site, d'autre part, la responsabilité respective de l'Homme et du Rat dans le maintien du foyer d'infection.

\section{Résultats}

\section{I. - Caractéristiques du site de transmission.}

Notre étude réalisée pendant le mois de novembre 1976 a pour cadre géographique le Bassin versant de Marigot-Beaugendre dans la commune de Vieux-Habitants en Basse-Terre. Cette vallée est drainée par la rivière Beaugendre qui alimente un réseau complexe de canaux d'irrigation s'étendant sur plus d'un kilomètre à travers des zones cultivées et parsemées d'habitations.

(1) Action concertée de la Délégation Générale à la Recherche Scientifique et Technique. Lutte biologique : Vecteurs. Coordonnateur : Pr Y.-J. Golvan. Recherches réalisées à la Station de Zoologie de 1'Institut National de la Recherche Agronomique. Petit-Bourg (Guadeloupe).

(2) Travail effectué dans le cadre d'une étude de système et de modélisation sous la direction du $\mathrm{P}^{r}$ J.-A. Rioux. 
Les recherches menées depuis février 1975 sur les densités cercariennes dans les cours d'eau et sur les effectifs de Biomphalaria glabrata ont montré que le canal étudié représente le principal site de transmission de toute la vallée; ce canal, branché sur un autre canal provenant directement de la rivière, est situé à $600 \mathrm{~m}$ de la prise d'eau (fig. 1) ; sa longueur totale est de 305 mètres.

Pour les besoins de notre étude et en tenant compte des habitations qui bordent ce canal, nous avons établi 10 stations d'analyses numérotées 0-1 à 9-10 dont les caractéristiques physiques sont résumées dans le tableau suivant :

Tableau I. - Caractéristiques physiques des différentes portions du canal.

\begin{tabular}{|c|c|c|c|c|c|c|c|c|c|c|}
\hline Portions & $0-1$ & $1-2$ & $2-3$ & 3-4 & $4-5$ & $5-6$ & $6-7$ & $7-8$ & $8-9$ & $9-10$ \\
\hline $\mathrm{m}$ & 3 & 3 & 30 & 40 & 3 & 5 & 40 & 45 & 30 & 30 \\
\hline 1 & 1,0 & 0,80 & 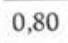 & 1,00 & 0,30 & T, & 0,60 & 0,80 & 0,80 & 2,00 \\
\hline leur $\mathrm{m}$ & 0,20 & 0,0 & 00 & 0,0 & 0,06 & 0,08 & 0.09 & 0 , & 0,08 & 0,40 \\
\hline Vitesse du courant $\mathrm{m} / \mathrm{s}$ & 0,18 & 0,1 & 0,18 & 0,1 & 0,20 & 0,30 & 0,20 & 0,2 & 0,18 & 0,01 \\
\hline Température à 12 & $23^{\circ} 5$ & $23^{\circ} 5$ & $23^{\circ} 5$ & $23^{\circ}$ & $23^{\circ}$ & $23^{\circ} 5$ & $23^{\circ} 5$ & $24^{\circ}$ & $25^{\circ}$ & $25^{\circ}$ \\
\hline
\end{tabular}

La portion 0-1 n'appartient pas au canal proprement dit mais représente une portion de 30 mètres, située en amont du branchement sur le canal principal ; elle est prise en considération afin de détecter un éventuel passage de cercaires du canal principal vers le canal étudié. Ce dernier est ombragé sur presque toute sa longueur (arbres à pain et cacaoyers). Les portions 4-5 et 5-6 sont de plus entièrement recouvertes par des plantations de "cannes brûlantes" (Dieffenbachia seguine). Après avoir traversé quelques "jardins créoles » le canal se perd dans un champ en flaques quasiment stagnantes $(0,01 \mathrm{~m} / \mathrm{s})$. Le débit moyen est environ de $40 \mathrm{~m}^{3} / \mathrm{h}$.

L'emplacement des habitations riveraines du canal, au nombre de 7 , est représenté sur la figure 1.

\section{II. - Données épidémiologiques.}

\section{1) Densités de vecteurs globales.}

L'étude quantitative des populations de B. glabrata le long du canal a été effectuée par un prélèvement exhaustif des mollusques dans chaque portion après asséchement complet du biotope. Au total, 2860 mollusques ont été récoltés. Les résultats de cette enquête sont résumés dans le tableau ci-dessous et la figure 2.

TABleau II. - Effectifs et densités du vecteur B. glabrata pour chaque portion du canal le 9 novembre 1976.

\begin{tabular}{|c|c|c|c|c|c|c|c|c|c|c|}
\hline Portions $\ldots .$. & $0-1$ & 1.2 & $2-3$ & $3-4$ & 4.5 & $5-6$ & $6-7$ & $7-8$ & 89 & $9-10$ \\
\hline fs de $B$. & 46 & 284 & 74 & 281 & 9 & 23 & 81 & 1067 & 160 & 165 \\
\hline Densités $B$. gla & 1,5 & 11,8 & 31,8 & 7,0 & 0,4 & 1,5 & 3,4 & 29,6 & 9,0 & 2,7 \\
\hline
\end{tabular}




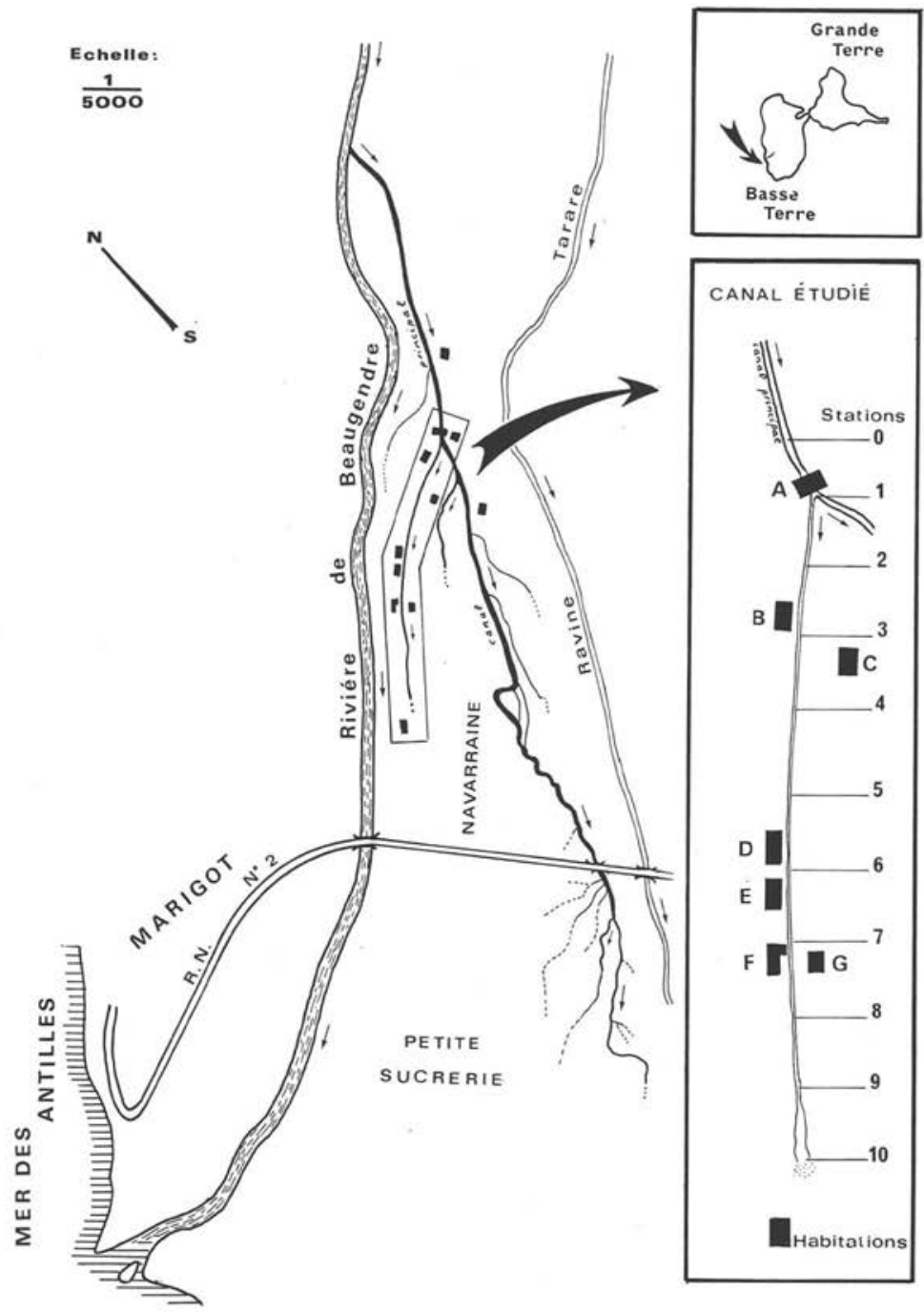

Fig. 1. - Cartographie du réseau de canaux dans la partie basse de la vallée de Beaugendre. Sectorisation et emplacement des habitations le long du canal étudié.

2) Densités de vecteurs parasités.

Les 2860 mollusques récoltés ont été testés individuellement au laboratoire; 349 d'entre eux émettaient des cercaires de $S$. mansoni, ce qui équivaut à une prévalence globale pour tout le canal de $12,2 \%$. La répartition des mollusques parasités est très inégale et les portions de la partie basse du canal sont de loin les plus contaminées (tableau III et fig. 2). 
TABLeAU III. - Effectifs, prévalences et densités des B. glabrata émettant des cercaires de $S$. mansoni le long du canal le 10 novembre 1976.

\begin{tabular}{|c|c|c|c|c|c|c|c|c|c|c|}
\hline Portions $\quad \ldots \ldots \ldots$ & $0-1$ & 1.2 & $2-3$ & $3-4$ & $4-5$ & $5-6$ & $6-7$ & 7.8 & $8-9$ & 9-10 \\
\hline $\begin{array}{l}\text { Effectifs de } B \text {. glabrata } \\
\text { positifs } \ldots \ldots \ldots \ldots \ldots \ldots\end{array}$ & 1 & 0 & 8 & 1 & 0 & 0 & 2 & 232 & 37 & 68 \\
\hline 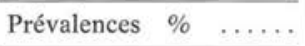 & 2,2 & 0 & 0,1 & 0,3 & 0 & 0 & 2,5 & 21,8 & 25,1 & 41,2 \\
\hline $\begin{array}{l}\begin{array}{l}\text { Densités de } \\
\text { positifs } / \mathrm{m}^{2}\end{array} \text { glabrata } \\
\ldots \ldots \ldots \ldots\end{array}$ & 0,03 & 0 & 0,30 & 0,02 & 0 & 0 & 0,08 & 6,40 & 2,10 & 1,10 \\
\hline
\end{tabular}

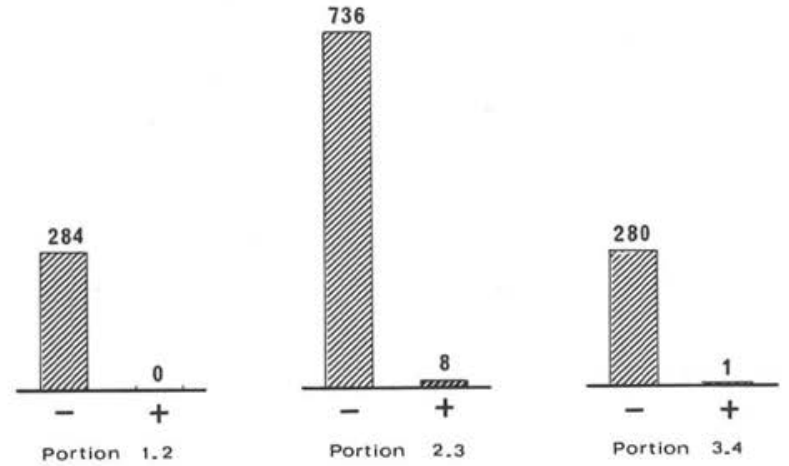

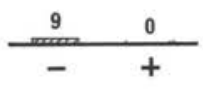

Portion 4.5

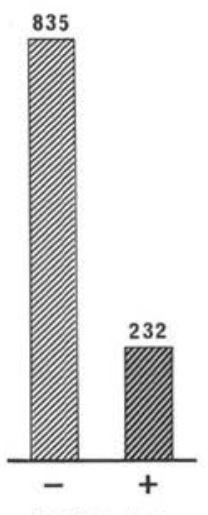

Portion 7.8

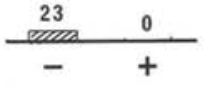

Portion 5.6

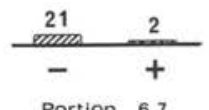

Portion 6.7

$$
\begin{aligned}
& \text { - B. glabrata sains } \\
& + \text { B. glabrata parasites }
\end{aligned}
$$

FIG. 2. - Répartition le long du canal des B.glabrata sains et parasités par S. mansoni.

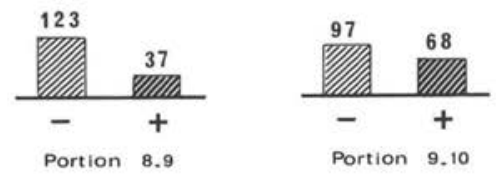



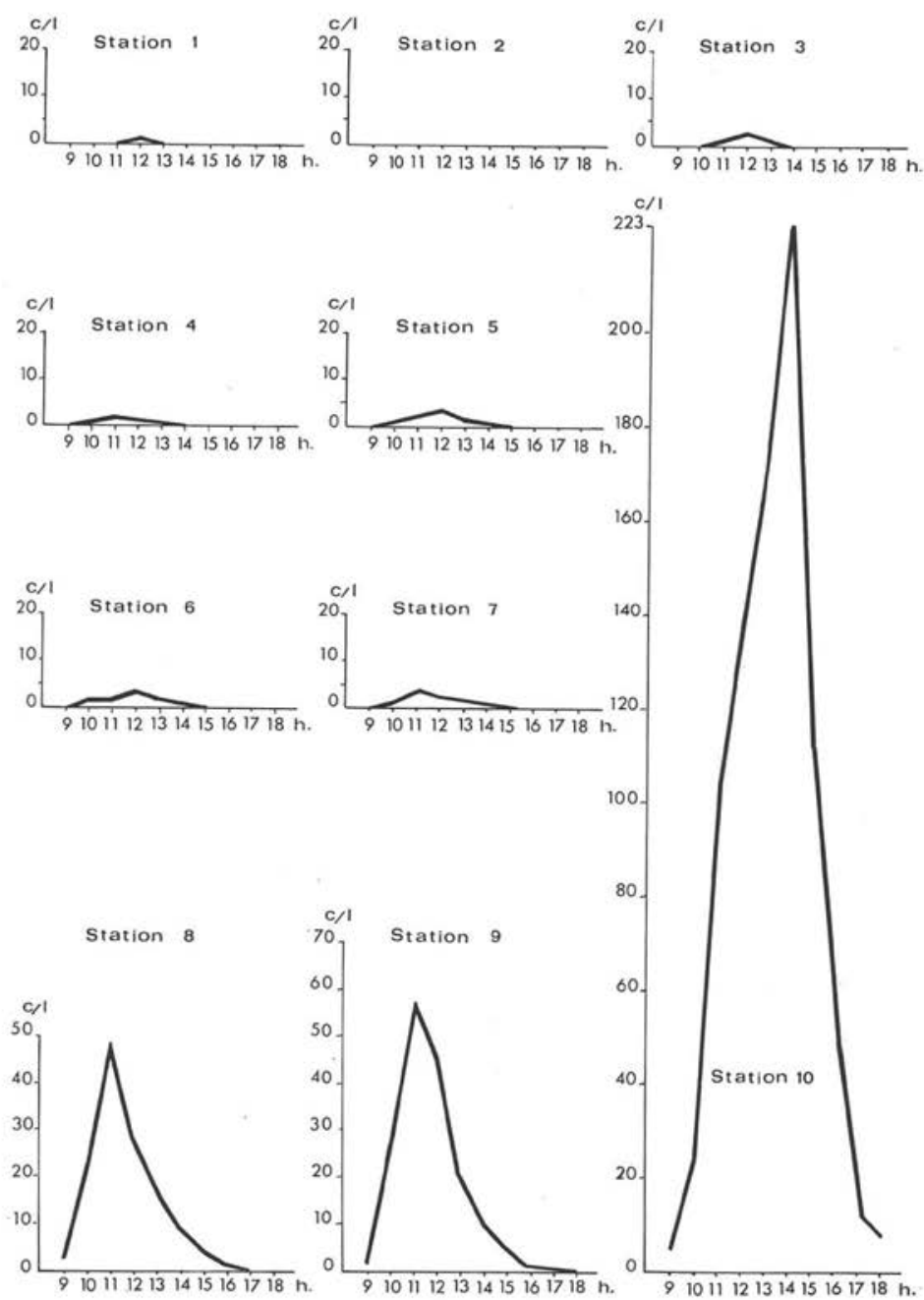

Fig. 3. - Rythmes de présence des cercaires de $S$. mansoni le long du canal étudié $(\mathrm{c} / 1=$ cercaires par litre $)$.

TABLEAU IV. - Densités cercariennes maximales de $S$. mansoni (cercaires/litre) le long du canal le 8 novembre 1976.

\begin{tabular}{|c|c|c|c|c|c|c|c|c|c|c|}
\hline Stations $\ldots \ldots \ldots \ldots \ldots$ & 1 & 2 & 3 & 4 & 5 & 6 & 7 & 8 & 9 & 10 \\
\hline Acrophase (h) $\ldots .$. & 11 & 11 & 12 & 11 & 12 & 12 & 11 & 11 & 11 & 14 \\
\hline Densités maximales $\mathrm{C} / 1$ & 0,4 & 0 & 2,6 & 2 & 3 & 2,8 & 3,2 & 48 & 57 & 223 \\
\hline
\end{tabular}




\section{3) Densités de Cercaires.}

Les densités de cercaires ont été évaluées en 10 points du canal (stations 1 à 10) par la technique de filtration directe d'échantillons d'eau de 10 litres (Rowan, 1958; Sandt, 1973 ; Houin et Golvan, 1975). Les échantillons sont prélevés à intervalles réguliers toutes les heures entre $8 \mathrm{~h}$ et $18 \mathrm{~h}$.

L'évolution temporelle des densités cercariennes est donnée pour chaque station à la figure 3. Elle se caractérise par un rythme de présence de type circadien avec une acrophase entre 11 et $12 \mathrm{~h}$ sauf accumulation des cercaires.

Nous reportons dans le tableau $I V$ la valeur des densités maximales et leur position dans la journée pour les 10 stations du canal.

\section{4) Distribution de la Schistosomose murine.}

Entre le 23 et le 27 novembre 1976, 29 rats (22 Rattus rattus et $7 R$. norvegicus) ont été capturés en bordure du canal. L'examen parasitologique des viscères révèle la présence de $S$. mansoni au niveau de l'intestin, du foie et des poumons de $10 \mathrm{R}$. rattus et de $2 R$. norvegicus (détermination P. Delattre). La répartition, portion par portion, des rats capturés et des rats bilharziens est donnée dans le tableau $V$; elle ne montre pas de concentration particulière des individus parasités.

Tableau V. - Répartition des Rats bilharziens le long du canal le 27 novembre 1976. ( $\mathrm{Rr}=$ Rattus rattus $; \mathrm{Rn}=$ Rattus norvegicus $)$.

\begin{tabular}{|c|c|c|c|c|c|c|c|c|c|c|}
\hline Portions $\ldots \ldots \ldots \ldots$ & $0-1$ & $1-2$ & $2-3$ & $3-4$ & $4-5$ & $5-6$ & 6-7 & $7-8$ & $8-9$ & $9-10$ \\
\hline Rats capturés $\ldots \ldots$ & - & $\begin{array}{l}1 \mathrm{Rr} \\
2 \mathrm{Rn}\end{array}$ & $4 \mathrm{Rr}$ & $4 \mathrm{Rr}$ & $2 \mathrm{Rr}$ & $\begin{array}{l}2 \mathrm{Rr} \\
1 \mathrm{Rn}\end{array}$ & 0 & $3 \mathrm{Rr}$ & $3 \mathrm{Rn}$ & $6 \mathrm{Rr}$ \\
\hline Rats parasités & - & $1 \mathrm{Rn}$ & $1 \mathrm{Rr}$ & $2 \mathrm{Rr}$ & $2 \mathrm{Rr}$ & $1 \mathrm{Rr}$ & 0 & 0 & $1 \mathrm{Rn}$ & $4 \mathrm{Rr}$ \\
\hline
\end{tabular}

\section{5) Distribution de LA Schistosomose humaine.}

De l'ensemble d'une enquête coprologique effectuée sur tous les habitants de la vallée de Beaugendre (J. A. Rioux, G. Lanotte, communication personnelle), nous reportons dans le tableau ci-dessous les résultats concernant uniquement les habitants riverains du canal.

Sur 32 personnes réparties en 7 habitations (fig. 1), 19 possèdent des œufs de $S$. mansoni dans les selles.

Tableau VI. - Effectifs et répartition des personnes bilharziennes riveraines du canal.

\begin{tabular}{|c|c|c|c|c|c|c|c|c|c|c|}
\hline Portions $\ldots \ldots \ldots \ldots \ldots$ & $1-2$ & $2-3$ & $3-4$ & $4-5$ & $5-6$ & 6-7 & $7-8$ & $8-9$ & $9-10$ & ГOtal \\
\hline Habitations $\quad \ldots \ldots \ldots \ldots$ & A & B & $\mathrm{C}$ & - & D & E & $\mathrm{F}+\mathrm{G}$ & - & - & 7 \\
\hline Habitants $\ldots \ldots \ldots \ldots$ & 4 & 5 & 6 & - & 8 & 1 & 8 & - & - & 32 \\
\hline Coprologie $(+)$ & 1 & 2 & 5 & - & 7 & 0 & 4 & - & - & 19 \\
\hline
\end{tabular}




\section{III. - Sectorisation d'après les données épidémiologiques.}

1) EN FONCTION DES POTENTIALITÉS DE TRANSMission.

La présence du Mollusque vecteur de la Schistosomose est effective sur toute l'étendue du biotope étudié mais sa répartition est très inégale suivant la portion de canal considérée. L'analyse quantitative des mollusques fait apparaître des portions "privilégiées " telles que 2-3 et 7-8 qui abritent respectivement 31,0 et 29,6 B. glabrata par $\mathrm{m}^{2}$ et des portions "défavorisées » telles que 4-5, 5-6 et 6-7 où les densités ne dépassent pas 3,4 B. glabrata par $\mathrm{m}^{2}$.

Les facteurs responsables de ces inégalités de la répartition des mollusques sont multiples et restent à déterminer avec précision. Nous pensons cependant, dans le cas précis de ce canal, que la présence dans les portions 2-3 et 7-8 d'une pollution permanente par des ordures ménagères favorise le développement et le maintien des importantes concentrations de vecteurs constatées à ces deux niveaux. Les mollusques y trouvent une source de nourriture abondante et appréciée (matières organiques, fruits, racines...). Pour les portions 4-5 et 5-6 à faible densité de vecteurs, nous pensons que le principal facteur limitant le développement des mollusques est la densité de la végétation de couverture (Dieffenbachia seguine) qui obscurcit entièrement ces portions du canal.

Cette hétérogénéité des densités vectorielles confère à chaque portion du canal une potentialité de transmission de la Schistosomose, différente suivant le nombre de mollusques qu'elle héberge :

En tenant compte des densités vectorielles (tableau II); nous pouvons regrouper les portions en 3 secteurs :

Secteur $A$ : compris entre les stations 1 et 4 , il abrite 1309 mollusques soit $14,87 / \mathrm{m}^{2}$; ce secteur doit être considéré comme un foyer potentiel important ;

Secteur $B$ : compris entre les stations 4 et 7 , il abrite 113 mollusques au total soit $1,76 / \mathrm{m}^{2}$; c'est un foyer potentiel faible ;

Secteur $C$ : compris entre les stations 7 et 10 dans la partie terminale du canal, il abrite 1392 mollusques soit $12,10 / \mathrm{m}^{2}$; c'est, comme le secteur A, un foyer potentiel important.

\section{2) EN FONCTION DE LA CONTAMINATION DU vecteur.}

Dans le secteur A, 9 mollusques seulement sur 1309 testés émettent des cercaires de $S$. mansoni, soit une prévalence de $0,69 \%$. Contrairement à ses potentialités, ce secteur du canal apparaît comme un site de faible transmission.

Dans le secteur B, 2 mollusques sur 113 sont positifs. La prévalence d'infestation est de $1,96 \%$, c'est encore une zone de faible transmission.

Dans le secteur C, 337 B. glabrata sur 1392 émettent des cercaires, soit une prévalence d'infestation de $24,2 \%$. Le secteur C représente le principal foyer de contamination du vecteur. A l'intérieur de ce secteur, la portion 7-8 prend une 
importance particulière puisqu'elle abrite à elle seule plus de $66 \%$ des mollusques parasités du canal (232/349).

On note donc des prévalences très différentes pour les secteurs $\mathrm{A}(0,69 \%)$ et $\mathrm{C}(24,2 \%)$ qui ne sont séparés que par une centaine de mètres et qui possèdent des effectifs de mollusques quasiment identiques $\left(14,87\right.$ et $12,10 \mathrm{~B}$. glabrata $\left./ \mathrm{m}^{2}\right)$.

La sectorisation du canal en fonction de la contamination des vecteurs fait apparaître l'extrême focalisation du site de contamination des mollusques puisque seul le secteur C (105 m de long) présente une prévalence d'infestation élevée.

\section{3) EN FONCTION DU RISQUE DE CONTAMINATION DES HÔTES VERTÉbrÉS.}

Nous considérons comme représentatives de chaque station la valeur des densités à l'acrophase (Théron et al., 1977). Ces densités peuvent être utilisées comme indicateur du risque de contamination des hôtes vertébrés. En nous référant au tableau $I V$, les secteurs A et B avec des densités de cercaires comprises entre 0 et $3,2 \mathrm{c} / 1$ apparaissent comme des sites à risque de contamination limité. Le secteur $\mathrm{C}$, au contraire, avec des densités de 48 à $223 \mathrm{c} / 1$ apparaît comme le site où le risque de contamination est le plus élevé. Ce secteur à haut risque se superpose étroitement au secteur de forte infestation des vecteurs. L'augmentation brutale du nombre de cercaires par litre entre la station $7(3,2 \mathrm{c} / 1)$ et la station $8(48 \mathrm{c} / 1)$ est bien entendu liée à l'accroissement du parasitisme des mollusques de la portion 7-8. Les densités de cercaires les plus élevées $(223 \mathrm{c} / 1)$ se situent dans la partie terminale du canal : l'explication est qu'à ce niveau l'importante diminution de la vitesse du courant qui passe de $0,18 \mathrm{~m} / \mathrm{s}$ (en 9) à $0,01 \mathrm{~m} / \mathrm{s}$ (en 10) provoque l'accumulation des cercaires émises sur place par les 68 B. glabrata positifs de la portion 9-10 et celles émises par les mollusques des portions $7-8$ et $8-9$ ce qui explique le chiffre record de $223 \mathrm{c} / 1$.

A cette évolution spatiale des densités cercariennes le long du canal, qui amène à considérer les parties basses comme les plus dangereuses, il faut superposer l'évolution temporelle de ces densités qui détermine entre 11 heures et 12 heures le risque le plus grand de contamination. Les filtrations horaires (fig. 3) confirment une fois de plus le rythme de présence caractéristique des cercaires de $S$. mansoni en milieu courant, à savoir une contamination des eaux à partir de 9 heures du matin, une acrophase entre 11 heures et 12 heures et une disparition des cercaires à partir de 16 heures le soir. Seule l'évolution cercarienne de la station 10 ne correspond pas à ce schéma. En effet, en ce point du canal, les densités maximales s'observent à 14 heures, et une quantité de cercaires supérieure à $10 \mathrm{c} / 1$ se maintient après 16 heures. C'est le ralentissement de la vitesse du courant $(0,01 \mathrm{~m} / \mathrm{s})$ qui, par accumulation des cercaires, retarde le pic cercarien et déplace à 14 heures le risque maximum de contamination (Théron et al., 1977).

\section{IV. - Responsabilité de l'Homme et du Rat dans l'infestation du vecteur.}

Nous pensons que les données que nous venons d'exposer (fig. 4) peuvent servir à une approche écologique des rôles respectifs de l'Homme et du Rat dans 
l'endémie bilharzienne. Du moins ces données permettent-elles de formuler une hypothèse sur l'origine de la contamination des vecteurs dans le biotope étudié.

Les densités de vecteurs et les densités d'hôtes vertébrés étant comparables dans les secteurs $\mathrm{A}$ et $\mathrm{C}$, on peut poser comme probable que les différences d'infestation entre mollusques de $\mathrm{A}$ et de C (fig. 4) ne peuvent être liées qu'à une différence dans le rejet de matières fécales contaminées. Si une telle différence existe, il semble difficile d'en rendre responsables les rats qui se déplacent tout le long du canal. Par contre, les conditions particulières à chacun des habitats humains font que suivant les cas, la pollution fécale peut avoir une intensité très différente.

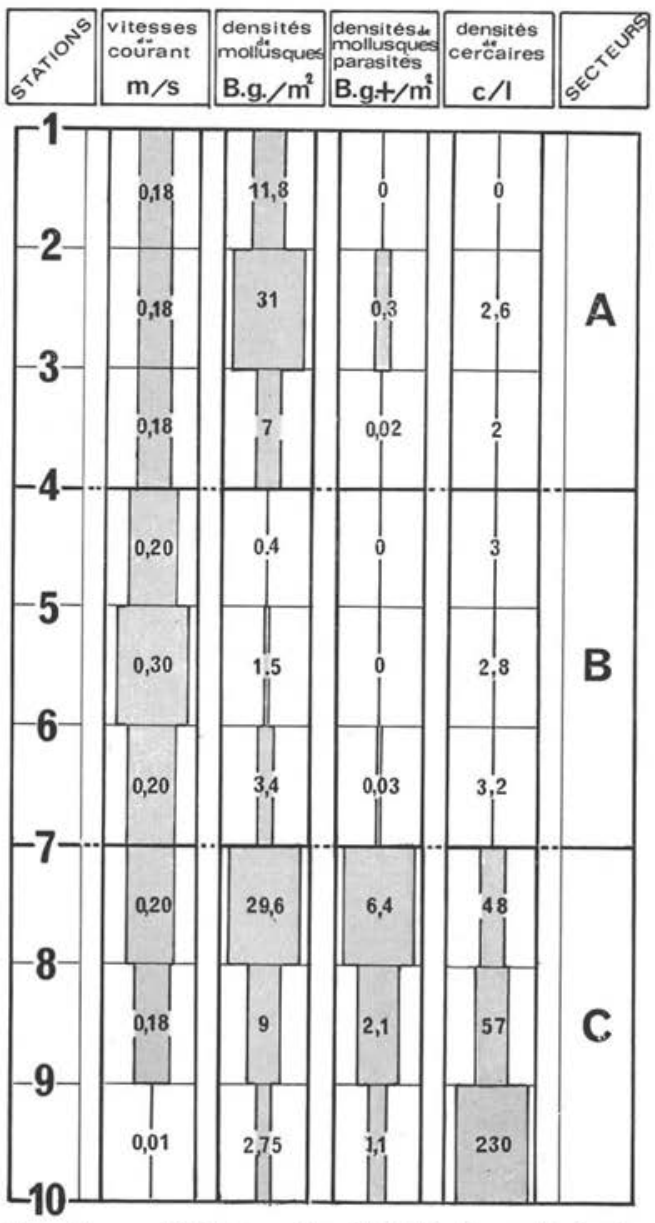

FIG. 4. - Tableau récapitulatif des principales données épidémiologiques concernant la transmission de $S$. mansoni le long du canal de Marigot.

Nos observations montrent précisément que sur les bords du canal étudié, une seule maison (habitation $\mathrm{F}$, fig. 1) est responsable d'un rejet direct et régulier dans le canal. Cette observation apporte une réponse à la question posée plus haut. En effet, si l'Homme est considéré comme responsable de la très forte infestation du secteur C, le Rat peut être tenu pour responsable au maximum du taux d'infestation très faible que l'on sbserve au secteur A.

Dans ces conditions, il apparaît que la responsabilité du Rat dans le fonctionnement d'un foyer de ce type est comparativement à celle de l'Homme, extrêmement réduite.

La contribution mineure du Rat à l'infestation des mollusques, nous conduit à penser que dans les canaux, la Schistosomose murine est tributaire de celle de l'Homme et que à la limite, le Rat est incapable d'y entretenir le cycle parasitaire.

Il semble d'ailleurs que dans un milieu stagnant comme le Grand Etang où l'habitat humain est nul mais où la quasi-totalité des rats est bilharzienne (Combes et al., 1975) le taux d'infestation des B. glabrata ne soit guère supérieure $(0,94 \%$, Nassi, 1977) à celui que nous observons au 
secteur A $(0,69 \%)$. Le maintien d'une endémie murine dans les conditions du Grand Etang peut alors s'expliquer par l'accumulation sur place des cercaires favorisant l'infestation des rats au crépuscule.

Deux remarques peuvent être ajoutées :

a) dans un site peu anthropisé comme le Grand Etang, le Rat a une fréquentation intense de l'eau puisqu'il y recherche sa nourriture comme l'atteste la Trématodose à Ribeiroia marini qu'il contracte en consommant des poissons (Nassi, 1977). Par contre, dans les canaux, les rats n'ont pas de poissons à consommer et se nourrissent préférentiellement dans les dépôts d'ordures autour des habitations ce qui limite leur fréquentation de l'eau ;

b) on peut envisager que l'infestation des rats ne soit pas le fait principalement du bain mais de la consommation des Planorbes. Cette proposition qui reste à démontrer expérimentalement serait en accord avec les innombrables coquilles de Planorbes consommés par les rats que nous avons trouvées aussi bien au bord même du canal de Marigot que sur les rives du Grand Etang. Nous pensons que cette consommation des Planorbes n'est pas le fait d'une capture sous l'eau mais qu'elle se produit lorsqu'une exondation quelconque rend les mollusques disponibles. Il faut remarquer que les biotopes où des rats ont été trouvés infestés en Guadeloupe (Combes et al. 1975 ; Nassi et al., 1975) sont précisément des milieux périodiquement asséchés (mise hors service des canaux, fluctuation du niveau du Grand Etang, asséchement saisonnier de la Mangrove).

\section{Conclusion}

L'étude des densités cercariennes et du taux de contamination des populations de $B$. glabrata dans la vallée de Beaugendre a permis de démontrer l'extrême focalisation des foyers à haut risque de contamination.

Notre étude montre que cette focalisation n'est pas déterminée par l'importance des populations de vecteurs puisque dans un même canal de dimensions modestes, des populations équivalentes de $B$. glabrata sont infestées de manières différentes.

Il apparaît par contre qu'une brusque inflation du taux de mollusques parasités et des densités de cercaires peut être reliée avec précision à une pollution fécale bien déterminée. La sectorisation du site de transmission étudié démontre en effet qu'une seule famille dont le type d'habitat entraîne une pollution fécale directe du cours d'eau fait passer les densités cercariennes de $3 \mathrm{c} / 1$ à $48 \mathrm{c} / 1$. Sachant que les filtrations de centaines de litres d'eau provenant de la rivière Beaugendre (1) n'ont jamais livré la moindre cercaire de $S$. mansoni, on peut interpréter les $3 \mathrm{c} / 1$ de l'eau du canal en amont de la famille contaminante comme un bruit de fond résultant soit d'une intervention des rats qui reste à évaluer, soit des défécations sauvages.

(1) $\mathrm{Ni}$ d'aucune autre rivière d'ailleurs en Guadeloupe. 


\section{REMERCIEMENTS}

Nous remercions M. P. Delattre (Station de Zoologie, Centre Antilles-Guyane de l'I.N.R.A.) qui a bien voulu, parallèlement au travail qu'il effectue sur le problème global des densités de rats et de leur contamination bilharzienne, capturer les exemplaires nécessaires à cette étude.

Nous remercions M. le $\mathrm{P}^{\mathrm{r}} \mathrm{J}$.-A. Rioux et $\mathrm{M}^{\mathrm{Hle}}$ le $\mathrm{D}^{\mathrm{r}}$ G. Lanotte (Faculté de Médecine de Montpellier) qui ont bien voulu nous communiquer les résultats de leur enquête sur l'infestation humaine dans la vallée de Beaugendre relatifs au secteur étudié.

\section{Bibliographie}

Combes (C.), Leger (N.) et Golvan (Y. J.), 1975. - Rats et Bilharziose en Guadeloupe. Acta Tropica., 32, 304-308.

Golvan (Y. J.), Combes (C.), Euzeby (J.), Houin (R.), Salvat (B.) et Lancastre (F.), 1974. Résultats préliminaires d'une enquête écologique sur Biomphalaria glabrata, vecteur de Schistosoma mansoni, en Guadeloupe. Proc. 3rd Int. Congr. Parasitol., vol. $I I, 821-822$.

Houin (R.), Golvan (Y.J.), Combes (C.), Deniau (M.) et Periac (P.), 1973. - Mollusques vecteurs de la Bilharziose en Guadeloupe. Approche physionomique des biotopes (étude préliminaire). Haliotis, 3, 73-79.

Houin (R.) et Golvan (Y. J.), 1975. - Field conditions of the transmission of Schistosoma mansoni in Guadeloupe (French West Indies). International Conference on Schistosomiasis. Cairo, Egypt. 35-36.

Nassi (H.), Lancastre (F.) et Porrot (J.), 1975. - Rat et bilharziose en Guadeloupe. Nouvelles données. Ann. Parasitol. hum. comp., 50, 731-733.

Nassi (H.), 1977. - Données sur le cycle biologique de Ribeiroia marini guadeloupensis n. ssp., Trématode stérilisant Biomphalaria glabrata en Guadeloupe. Entretien du cycle en vue d'un contrôle éventuel des populations de mollusques. Acta Tropica, (sous presse).

Rowan (W.B.), 1958. - Daily periodicity of Schistosoma mansoni cercariae in Puerto Rican waters. Ann. Trop. Med. Hyg., 7, 374-381.

SANDT (D. G.), 1973. - Direct filtration for recovery of Schistosoma mansoni cercariae in the field. Bull. Wld. Hlth. Org., 48, 27-34.

Theron (A.), Pointier (J.-P.) et Combes (C.), 1977. - Recherches sur les facteurs responsables des densités de cercaires de Schistosoma mansoni en Guadeloupe. Ann. Parasitol. hum. comp., 52, 421-433. 